\title{
Statistical Analysis of Structural Brain Connectivity
}

\author{
Renske de Boer ${ }^{1,2}$, Michiel Schaap ${ }^{1}$, Fedde van der Lijn ${ }^{1}$, Henri A. Vrooman ${ }^{1}$, \\ Marius de Groot ${ }^{1}$, Meike W. Vernooij ${ }^{2,3}$, M. Arfan $\mathrm{Ikram}^{2,3}$, \\ Evert F.S. van Velsen ${ }^{1,2}$, Aad van der Lugt ${ }^{3}$, \\ Monique M.B. Breteler ${ }^{2}$, and Wiro J. Niessen ${ }^{1,4}$ \\ 1 Biomedical Imaging Group Rotterdam, Departments of Radiology \& \\ Medical Informatics, Erasmus MC, Rotterdam, The Netherlands \\ 2 Department of Epidemiology, Erasmus MC, Rotterdam, The Netherlands \\ 3 Department of Radiology, Erasmus MC, Rotterdam, The Netherlands \\ 4 Imaging Science \& Technology, Faculty of Applied Sciences, Delft University of \\ Technology, Delft, The Netherlands
}

\begin{abstract}
We present a framework for statistical analysis in large cohorts of structural brain connectivity, derived from diffusion weighted MRI. A brain network is defined between subcortical gray matter structures and a cortical parcellation obtained with FreeSurfer. Connectivity is established through minimum cost paths with an anisotropic local cost function and is quantified per connection. The connectivity network potentially encodes important information about brain structure, and can be analyzed using multivariate regression methods. The proposed framework can be used to study the relation between connectivity and e.g. brain function or neurodegenerative disease. As a proof of principle, we perform principal component regression in order to predict age and gender, based on the connectivity networks of 979 middle-aged and elderly subjects, in a 10-fold cross-validation. The results are compared to predictions based on fractional anisotropy and mean diffusivity averaged over the white matter and over the corpus callosum. Additionally, the predictions are performed based on the best predicting connection in the network. Principal component regression outperformed all other prediction models, demonstrating the age and gender information encoded in the connectivity network.
\end{abstract}

\section{Introduction}

Both functional and anatomical connectivity of the brain are areas of increasing research interest. Functional connectivity is mainly established through functional magnetic resonance imaging (fMRI) while diffusion MRI has been applied for assessing anatomical or structural connectivity. Both types have been analyzed by modeling connectivity as a complex network and applying graph theory approaches to study the network topology. A review of graph theoretical analysis of complex brain networks is given in [1]. 
In structural connectivity the network nodes represent brain regions and the connections are usually established through probabilistic or streamline tractography. Streamline tractography might be incapable of finding a connection in regions of uncertain directionality, due to e.g. crossing fibers or noise. To overcome this problem, probabilistic tractography was proposed in which multiple flow vectors are chosen from a distribution around the principal eigenvector of the diffusion tensor, e.g. [23]. Alternatively, a connection between two regions can be established through directional dependent minimum cost path methods [4 5 : 6 6 ]. These methods compute the minimum cost to get from a start region to another point in the image. The costs depend on both location and direction and can be defined based on the diffusion MRI data. Even though minimum cost path methods are closely related to probabilistic tractography, they contain no random factor and will therefore give reproducible results. This is an advantage when creating a connectivity network. Furthermore, minimum cost path methods find the globally optimal paths, except for some inaccuracy due to discretization. Probabilistic tractography is more likely to end at a local optimum because of limited flow vector sampling in a modeled distribution.

We present a new framework for statistical analysis of structural brain connectivity based on minimum cost paths. The connectivity network is established from diffusion weighted images (DWI) using the method previously proposed by Melonakos et al. 7]. The network nodes are based on segmentations and cortical parcellations obtained using FreeSurfer [8]. By quantifying the connectivity between the nodes, we construct a mean connectivity brain network that can be analyzed using multivariate statistics. The results can be used to study connectivity changes in e.g. aging, cognitive decline, and neurological or psychiatric disorders. As a proof of principle, we perform principal component regression in order to predict age and gender in a large dataset of aging subjects. We compare the results to predictions based on fractional anisotropy (FA) and mean diffusivity (MD) averaged over the white matter and the corpus callosum, and prediction based on the best predicting connection.

\section{Framework}

\subsection{Connectivity}

Minimum cost paths. The connectivity between two brain regions is defined by minimum cost paths. Start region $R$ is connected with point $\mathbf{p}$, through the path $\Gamma$ with minimum cumulative traveling cost. This cumulative cost $u(\mathbf{p})$ is given by

$$
u(\mathbf{p})=\min _{\Gamma} \int_{0}^{L} \psi\left(\mathbf{x}(s), \mathbf{x}^{\prime}(s)\right) d s
$$

where $s$ is the arc length along $\Gamma ; L$ is the length of $\Gamma ; \mathbf{x}(s)$ is the position on the path; $\mathbf{x}^{\prime}(s)=\frac{\nabla u}{\|\nabla u\|}$ is the unit local direction of the path; and $\psi(\mathbf{x}, \mathbf{v})$ is the local anisotropic cost function, defining the cost at position $\mathbf{x}$ in direction $\mathbf{v}$. 
Local Cost Function. In order to have the minimum cost paths run through white matter bundles, a local cost function which is low on, and in the direction of, white matter tracts should be defined. To this end, different cost functions have been proposed. Some are based on the diffusion tensor model 46]. These methods are not suitable for modeling regions of multiple fiber populations e.g. in the event of crossing fibers. We choose a local cost function based on the set of acquired diffusion weighted images [5] and decide to use the local cost function proposed in [7].

$$
\psi(\mathbf{x}, \mathbf{v})=\left(\frac{S(\mathbf{x}, \mathbf{v})}{\int_{\mathbf{w} \perp \mathbf{v}} \frac{S(\mathbf{x}, \mathbf{w})}{S(\mathbf{x}, \mathbf{0})} d \mathbf{w}}\right)^{3}
$$

Where $S(\mathbf{x}, \mathbf{v})$ is the (interpolated) DWI value at position $\mathbf{x}$ and (interpolated) direction $\mathbf{v} . S(\mathbf{x}, \mathbf{0})$ is the value of the image without diffusion weighting (B0) at position $\mathbf{x} . S(\mathbf{x}, \mathbf{v})$ is low if the diffusion at position $\mathbf{x}$ in direction $\mathbf{v}$ is high, because of diffusion-related signal loss. Therefore, the costs are low if the diffusion is high in direction $\mathbf{v}$ compared to perpendicular directions.

Quantifying Connectivity. For every connection a value needs to be obtained depicting the connectivity between the connected nodes. It is possible to use different measures for quantifying connectivity. Equation 1 can be generalized to integrate any local measure, $f(\mathbf{x})$, from the start region $R$ to point $\mathbf{p}$ over the minimum cost path, defined by $\psi(\mathbf{x}, \mathbf{v})$. Dividing the cumulative measure by the path length $L$ yields a mean measure, $\bar{f}(\mathbf{x})$, over the minimum cost path.

$$
\bar{f}(\mathbf{p})=\frac{1}{L} \int_{0}^{L} f(\mathbf{x}(s)) d s
$$

In this way it is possible to calculate e.g. the mean FA or mean MD over the minimum cost path. Although these measures are based on a tensor model, which has disadvantages as discussed before, the local cost function is not, and depends on both local anisotropy and diffusion. We use the mean cost, obtained by dividing cumulative cost by path length, as connectivity measure. Dividing by the length of the minimum cost path is necessary in order to correct for differences in head size and/or brain atrophy.

\subsection{Construction of the Connectivity Network}

To enable statistical analysis of brain connectivity maps, corresponding subcortical and cortical regions should be defined in all subjects. Hereto, the publicly available FreeSurfer software is used, which is capable of segmenting subcortical structures [8] and parcellating the cortex [9]. T1-weighted (T1w) scans are given as input for the FreeSurfer reconstruction pipeline. The resulting segmentation and cortical parcellation are transformed according to rigid registration of the T1w scan to the B0 diffusion image performed by Elastix 10. Minimum cost paths are calculated as proposed by Melonakos et al. 7] and only performed within gray and white matter as defined by the FreeSurfer segmentation. 
From the FreeSurfer segmentation, we use 17 subcortical structures succeedingly as start regions: the brain stem, and the left and right segmentations of thalamus, caudate nucleus, putamen, pallidum, hippocampus, amygdala, accumbens area and ventral diencephalon. As target regions we use the FreeSurfer cortical parcellation based on the Destrieux 2009 atlas, which divides the cortex in 75 regions per hemisphere, augmented by the 16 subcortial regions not currently used as start region. The connectivity between start and target region is represented by the minimum of the mean cost among all voxels in the target region. The resulting connectivity network consists of $2 \times 75+17=167$ nodes and $n=(167-1) \times 17=2822$ connections, which for $m$ subjects combines into a $m \times n$ connectivity matrix.

\subsection{Statistical Analysis}

The connectivity matrix can be used to investigate changes in connectivity with e.g. normal aging, in cognitive decline, and in psychiatric disorders. As a proof of principle, we perform principal component regression (PCR) in an attempt to predict age and gender of our test subjects. For PCR we perform principal component analysis on the connectivity matrix. The first 15 principal components are used as input for multivariate linear regression to predict age and for multivariate logistic regression to predict gender. We compare our results to predictions based on five different univariate regression models. Two models are based on diffusion measures in the entire white matter, namely mean FA and MD (WM-FA and WM-MD). Additionally, two predictions are based on the regional measures of mean FA and mean MD in the corpus callosum (CC-FA and CC-MD). White matter and corpus callosum are defined by the FreeSurfer segmentation. For the fifth model, we perform a regression for every connection in the connectivity network. The connection with the smallest root mean squared deviation based on the training set, is used for the final prediction.

All experiments are performed in a 10-fold cross validation. For every fold, the regression coefficients are estimated on the training set and the prediction is evaluated on the test set. The alternative prediction models use the same subdivision of subjects for the cross validation. Age prediction is evaluated by mean absolute difference $(|\Delta|)$ between predicted and actual age. For the prediction of gender, the percentage of correctly predicted subjects is reported.

In population studies of the elderly, gender may not be distributed evenly over all ages. In that case, if both age and gender relate to the prediction model variable, it is necessary to correct for one when predicting the other. This correction is performed by linear regression with the confounding variable as input, and the model variable as output. The residuals are used for prediction.

\section{Results}

Imaging data from the Rotterdam Scan Study [11, acquired in 2005-2006, were used for the evaluation of the method. Scans were obtained on a $1.5 \mathrm{~T}$ GE scanner using an 8-channel head coil. The DWI scanning protocol had a $b$-value 

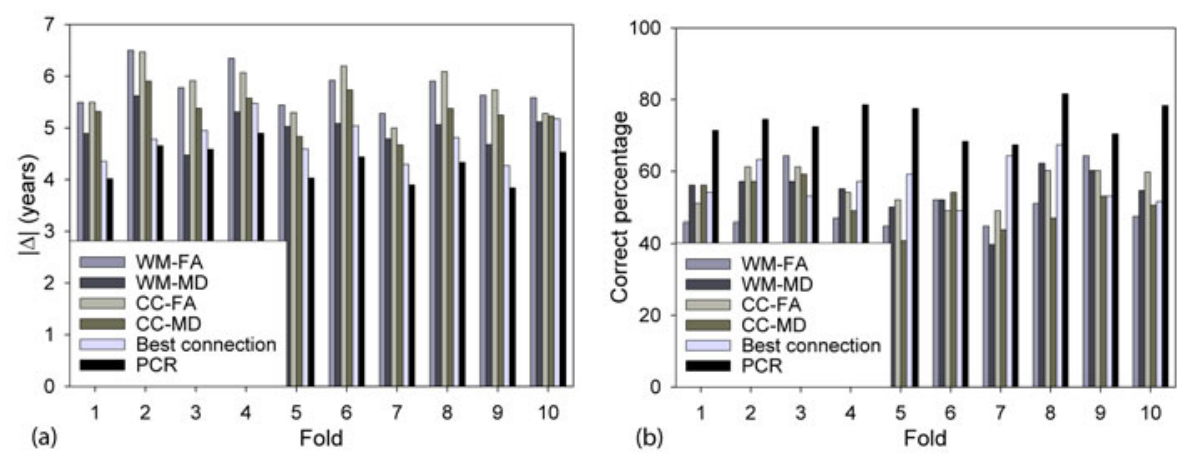

Fig. 1. Mean absolute difference in age prediction (a) and percentage correctly predicted gender (b) for all models at all folds

of $1000 \mathrm{~s} / \mathrm{mm}^{2}$ in 25 non-collinear directions, and one volume was acquired without diffusion weighting. Voxel sizes were $0.8 \times 0.8 \times 3.5 \mathrm{~mm}$. Head motion and Eddy current corrections of the DWI were performed with FDT, part of FSL[12]. FDT was also used to fit the tensor for calculation of the FA and MD images. The 3D T1w images had voxels sizes of $0.49 \times 0.49 \times 0.8 \mathrm{~mm}$.

Subjects with cortical infarcts, artifacts in any of their scans or FreeSurfer errors were excluded from the analysis. The remaining 979 subjects had a mean age of $68.5 \pm 7.4$ years (range 59.0 - 96.7) and consisted of 469 men and 510 women.

Figure 1a shows the age prediction error for the different regression models per fold. Lower $|\Delta|$ indicates better results. The PCR model resulted in the lowest $|\Delta|$ for nine out of ten folds, while the FA-based models showed overall the highest $|\Delta|$. Table 1 shows the results averaged over all folds. PCR on the connectivity matrix improved age prediction with at least 0.7 years compared to the global and regional measures. It also showed an improvement of 0.5 years compared to the best predicting single connection. The 15 principal components explained on average $65.8 \%$ of the variance in the gender corrected data.

Figure $1 \mathrm{~b}$ shows the gender prediction accuracy for all models and folds. PCR outperformed the other models on all folds. Table 1 shows that, while the prediction based on the global and regional diffusion measures was close to random, PCR on the connectivity matrix obtained a gender prediction accuracy of $74.1 \%$. The 15 principal components explained on average $61.6 \%$ of the variance in the age corrected data.

Figure 2 shows the connections running between the right putamen start region and all target regions in one subject. These connections are obtained by tracing back in the direction of the lowest cumulative cost, starting at the target voxel. By combining the 15 principal components with the PCR coefficients, it is possible to obtain the per connection regression coefficients. The connections in Fig. 2 are colored according to their regression coefficient for gender prediction as calculated based on the training set of the tenth fold. 
Table 1. Per model, mean absolute difference in years in predicted age and percentage correctly predicted gender averaged over all folds

\begin{tabular}{lcc}
\hline & $\begin{array}{c}\text { Age prediction } \\
|\Delta| \text { (years) }\end{array}$ & $\begin{array}{c}\text { Gender prediction } \\
\text { Correct }(\%)\end{array}$ \\
\hline WM-FA & 5.8 & 50.8 \\
WM-MD & 5.0 & 54.4 \\
CC-FA & 5.8 & 55.8 \\
CC-MD & 5.3 & 51.1 \\
Best connection & 4.8 & 57.2 \\
PCR & 4.3 & 74.1 \\
\hline
\end{tabular}

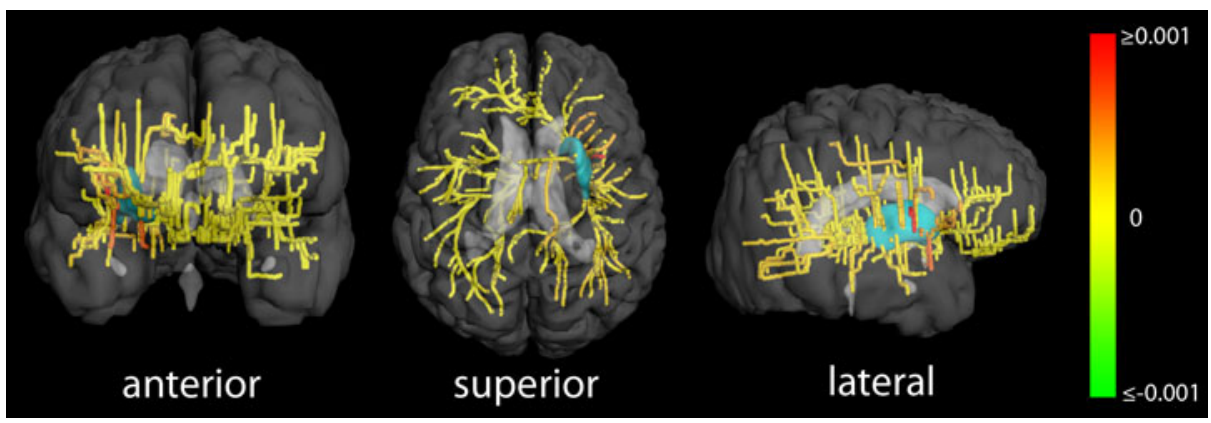

Fig. 2. Subject specific connections starting from the right putamen (in cyan). Connections are colored according to their regression coefficients for gender prediction.

\section{Conclusion and Discussion}

We present a new framework for structural connectivity analysis in large datasets of diffusion weighted brain MRI. Connectivity is established through minimum cost paths with an anisotropic local cost function based on DWI. Using brain regions defined by FreeSurfer, a connectivity network is obtained that can be analyzed using multivariate regression methods. As a proof of principle we performed PCR in order to predict age and gender. WM-FA and WM-MD are capable of predicting age with a mean absolute difference of 5.8 and 5.0 years respectively. PCR of the connectivity matrix reduces the prediction error to 4.3 years, suggesting that some of the network connections contain more information regarding age than others. The tight age distribution of the subjects makes prediction a difficult task. Predicting the mean age or, in case of a skewed distribution, the median age can already be quite accurate in tight distributions. The mean absolute difference for prediction of the mean age of the training set is 6.0 years; the median age reduces this difference to 5.6 years. Both results are close to the results of the global and regional diffusion measure models, but PCR 
shows a clear improvement. On gender prediction, PCR improves the correctly predicted percentage from close to random for the global and regional diffusion measures to $74.1 \%$.

PCR predicts more accurate than the best predicting connection for both age and gender. It could be argued that this improvement is caused by the use of 15 versus one regression variable, or by the aggregation of connections into groups. The latter could result in averaging of noise due to redundancy in the data. To test the additional advantage of using principal components over using the mean of groups of connections, we randomly assigned all connections into 15 groups, which are used as input for multivariate linear regression. This model results in a mean absolute difference in predicted age of 4.9 years and a percentage of correctly predicted gender of $62.7 \%$. These results are comparable or better than the best predicting connection. However, the results are not better than the PCR results, which shows the benefits of the use of principal components.

Minimum cost path methods will always find a connection between two regions. This is an advantage when constructing a connectivity matrix in which corresponding connections need to be found in all subjects. As a result, the proposed framework differs from existing approaches in that the analysis is carried out on all connections instead of a subset. Furthermore, there is no need for parameters that determine which connections are retained in the analysis [1. The disadvantage, however, is that it might suggest biologically implausible direct connections between regions. It is important to keep in mind that the connections that are found may also represent indirect connections, connecting two regions through a third.

Finally, the presented whole-brain analysis method is especially suited to study differences in connectivity in populations, where previously proposed tractography-based analyses are mainly directed at studying the topology of the structural network. Robinson et al. also perform statistical analysis of brain connectivity, but they use probabilistic tractography based on a tensor model and classify their subjects in two wide range age groups [13. In [14 they perform their tractography based on a two-component partial volume model and classify in two strongly contrasting age groups. We do not fit any model to the DWI data and predict age as a continuous variable. The prediction of age or gender of a person is of course not very relevant in research and clinical practice. The performed experiment can, however, be used to assess which connections have the largest contribution to the prediction. These connections contain the most information regarding age or gender. In future work, we will add the cortical parcellation regions as start regions, creating a connectivity network that includes cortico-cortical connections.

In conclusion, we present a framework for construction of a structural brain connectivity network that potentially can be used to study brain changes in e.g. aging, neurodegenerative disease or psychiatric disorders. As a proof of principle, we perform PCR in order to predict age and gender based on a network of connections between subcortical structures and cortical regions. PCR outperforms 
the predictions based on global and regional averaged FA and MD and the best predicting single connection, demonstrating the value of the information encoded in the connectivity network.

\section{References}

1. Bullmore, E., Sporns, O.: Complex brain networks: graph theoretical analysis of structural and functional systems. Nat. Rev. Neurosci. 10, 186-198 (2009)

2. Behrens, T.E.J., Woolrich, M.W., Jenkinson, M., et al.: Characterization and propagation of uncertainty in diffusion-weighted MR imaging. Magn. Reson. Med. 50, 1077-1088 (2003)

3. Parker, G.J.M., Haroon, H.A., Wheeler-Kingshott, C.A.M.: A framework for a streamline-based probabilistic index of connectivity (PICo) using a structural interpretation of MRI diffusion measurements. J. Magn. Reson. Imaging 18, 242-254 (2003)

4. Jackowski, M., Kao, C.Y., Qiu, M., Constable, R.T., Staib, L.H.: White matter tractography by anisotropic wavefront evolution and diffusion tensor imaging. Med. Image Anal. 9, 427-440 (2005)

5. Pichon, E., Westin, C.F., Tannenbaum, A.R.: A Hamilton-Jacobi-Bellman approach to high angular resolution diffusion tractography. In: Duncan, J.S., Gerig, G. (eds.) MICCAI 2005. LNCS, vol. 3749, pp. 180-187. Springer, Heidelberg (2005)

6. Fletcher, P.T., Tao, R., Jeong, W.K., Whitaker, R.T.: A volumetric approach to quantifying region-to-region white matter connectivity in diffusion tensor MRI. Inf. Process Med. Imaging 20, 346-358 (2007)

7. Melonakos, J., Pichon, E., Angenent, S., Tannenbaum, A.: Finsler active contours. IEEE Trans. Pattern Anal. Mach. Intell. 30, 412-423 (2008)

8. Fischl, B., Salat, D.H., van der Kouwe, A.J.W., et al.: Sequence-independent segmentation of magnetic resonance images. NeuroImage 23(suppl. 1), S69-S84 (2004)

9. Fischl, B., van der Kouwe, A., Destrieux, C., et al.: Automatically parcellating the human cerebral cortex. Cereb. Cortex 14, 11-22 (2004)

10. Klein, S., Staring, M., Murphy, K., Viergever, M.A., Pluim, J.: Elastix: A toolbox for intensity-based medical image registration. IEEE Trans. Med. Imaging 29, 196205 (2010)

11. Hofman, A., Breteler, M.M.B., van Duijn, C.M., et al.: The Rotterdam Study: 2010 objectives and design update. Eur. J. Epidemiol. 24, 553-572 (2009)

12. Woolrich, M.W., Jbabdi, S., Patenaude, B., et al.: Bayesian analysis of neuroimaging data in FSL. NeuroImage 45, S173-S186 (2009)

13. Robinson, E.C., Valstar, M., Hammers, A., Ericsson, A., Edwards, A.D., Rueckert, D.: Multivariate statistical analysis of whole brain structural networks obtained using probabilistic tractography. In: Metaxas, D., Axel, L., Fichtinger, G., Székely, G. (eds.) MICCAI 2008, Part I. LNCS, vol. 5241, pp. 486-493. Springer, Heidelberg (2008)

14. Robinson, E.C., Hammers, A., Ericsson, A., Edwards, A.D., Rueckert, D.: Identifying population differences in whole-brain structural networks: A machine learning approach. NeuroImage, 910-919 (2010) 